\title{
Multidisciplinary Design Optimization of a Deorbit Maneuver Considering Propulsion, TPS, and Trajectory
}

\author{
Amirhossein Adami \\ Amirkabir University of \\ Technology, PhD student \\ Hafez street, Tehran, Iran
}

\author{
Mahdi Mortazavi \\ Amirkabir University of \\ Technology, \\ Hafez street, Tehran, Iran
}

\author{
Mehran Nosratollahi \\ Space Research Institute \\ $24^{\text {th }}$ kilometer of Tehran-Karaj \\ highway, Tehran, Iran
}

\begin{abstract}
Unguided reentry capsules are usually involved in ballistic entry. The final states (such as altitude and velocity of parachute activity) depend on initials parameters. Reentry trajectory parameters differently affect the thermal protection system (TPS), required deorbit propellant, and structural load. The purpose of this paper is to optimal design of deorbit parameters to minimize the thermal protection system mass and deorbit propellant mass using multidisciplinary design optimization technique. Genetic Algorithm (GA) is used to simultaneously optimize TPS discipline, propulsion discipline and trajectory in present of the all trajectory and configuration constraints. To do this, every discipline is mathematically modeled. A suitable framework of Multidisciplinary design optimization (MDO) is developed. Then, the reentry mission is optimized according to the each discipline. The results show that simultaneous optimization is more efficient in comparison with single discipline optimization such as TPS optimization or deorbit propulsion system (propulsion and propellant) optimization.
\end{abstract}

\section{Keywords}

Reentry Vehicle, Deorbit Maneuver, Optimization, MDO, Genetic Algorithm

\section{INTRODUCTION}

Optimization of a complex system is always involved in optimization of more than one objective. MDO techniques can be widely used for reentry mission because many disciplines are used. In addition, MDO techniques have been developed to reach a global optimum solution. Reusable launch vehicles, manned reentry capsules, interplanetary vehicles and etc. are the example of reentry missions. Reentry mission is a very complex and a computationally intensive problem. The optimization of such a complex mission is still a formidable computational challenge due to very large CPU power and memory requirements. Therefore, it is better to divide the reentry mission into the some different disciplines and each discipline is optimized according to the system level optimizer. As a state of the art following works can be introduced.

Single discipline optimization is more found in literature [1; $2 ; 3 ; 4 ; 5 ; 6 ; 7]$ but as mentioned before, single disciplines optimization do not guarantee to find the global optimum solution.

In reference [8] the optimal design of a reentry vehicle configuration to minimize the heat absorbed (thermal protection system mass) and structural mass is carried out by a modified Genetic Algorithm and results are compared with multi-objective Genetic Algorithm.
Reference [9] introduced the method to optimum design of a controllable reentry capsule for minimum landing velocity using MDO technique. Structure, aerodynamics, TPS and simulation disciplines was used.

Multidisciplinary design optimization of a manned reentry mission was introduced in reference [10] considering trajectory, structure, TPS, and aerodynamic configuration. AAO framework was used.

According to the above researches, it can be understood that atmospheric reentry is more interesting. However, reentry phase depends on the deorbit phase. Deorbit phase and reentry phase demand different conditions and conflict together. This paper focuses on planner deorbit considering propulsion system, TPS and trajectory to tradeoff between the atmospheric phase and reentry phase.

The total mass is a suitable estimation of every reentry mission cost. If deorbit maneuver is separated from atmospheric phase then, aerodynamic disciplines is limited by using a fix configuration. It means that reentry mission breaks down to the two important phase including deorbit and atmospheric reentry. If aerodynamic configuration was limited; therefore, heat shield mass, structural mass, propulsion mass and required deorbit propellant mass are the most part of the total mass that should be minimized.

The TPS mass depends on configuration and heat loads which are time dependent. The structural mass depends on configuration and aerodynamic forces. The propulsion mass depends on trajectory (deorbit velocity) and empty RV mass (TPS mass, payload mass and structural mass).

The propellant mass conflicts with TPS mass and flight time (final error). These conflicts can be solved by multidisciplinary design optimizations (MDO) techniques such as all at once (AAO), bi-level integrated system synthesis (BLISS), collaborative optimization (CO) and etc. In this paper, every discipline is developed based on AAO framework because of hard coupling of disciplines and time variable of some parameters. All-at-Once is the most basic MDO technique and has wide industry acceptance [8]. In $\mathrm{AAO}$, control is given to a system-level optimizer that ensures a global objective is met by having a single designer control the entire system. AAO solves the global MDO problem by moving all local-level design variables and constraints away from each discipline to a new system-level optimizer entrusted with optimizing a global objective.

For a fixed configuration, maximum allowable g-load and aerodynamic coefficients (varying by configuration) are constant and the available propellant volume is limited. An unguided reentry capsule is usually involved in ballistic entry and final states (such as altitude and velocity of parachute 
action) depend on reentry parameters. Therefore, final error depends on reentry trajectory parameters. The earth rotating plays a critical role in final error especially if planner deorbit has been considered. Mathematical model of each discipline are shortly introduced at follow.

\section{CONFIGURATION AND FINAL POSITION CONSTRAINTS}

The general bi-conic configuration is selected for optimization. This configuration has a good aerodynamic stability and it is suitable for unguided reentry missions [10].
Heat shield thicknesses are introduced in figure (1). The geometry parameters selected as same as reference [10].

The preliminary orbit and related parameters are selected as same as reference [10]. Final reentry situation are selected as, $50^{\circ} \mathrm{E}, 40^{\circ} \mathrm{N}$ and altitude equals to $5 \mathrm{~km}$. Mach number should be below than 3 and final position error should be kept lower than $1 \mathrm{~km}$.

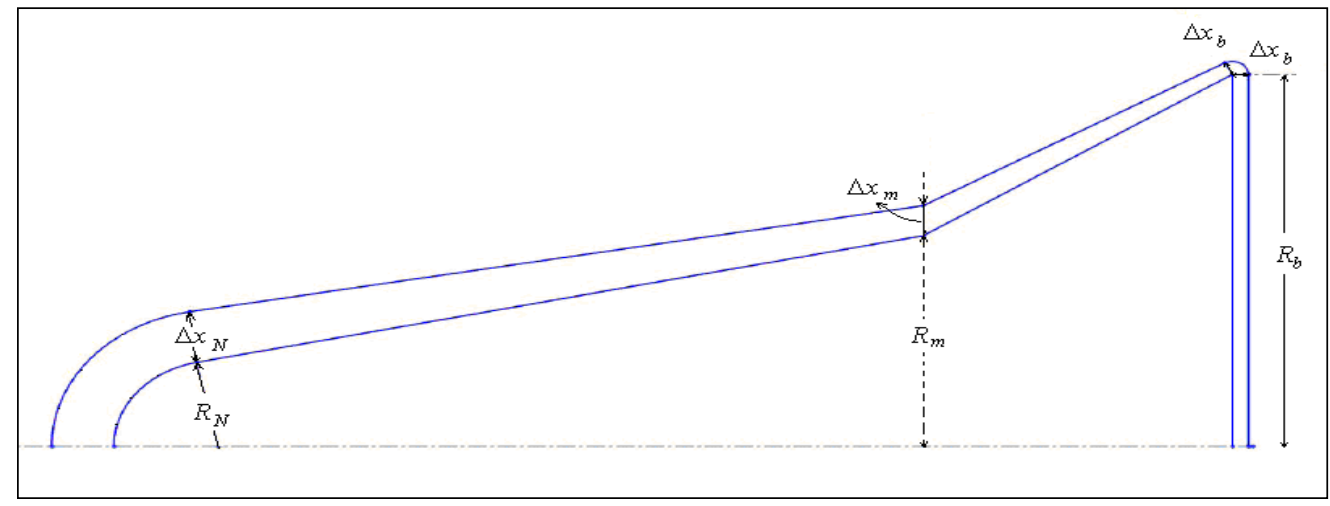

Figure 1: The geometry and TPS parameters

\section{TRAJECTORY SIMULATIONS}

Spherical earth configuration and standard atmosphere are considered for 3D trajectory simulation. Ballistic trajectory without any guidance and control leads to planar analysis so, the trajectories are considered in the orbit plane. The symmetric configuration and aerodynamic stability lead to zero angle of attack $(\alpha)$ at dense atmosphere and so lift is negligible. The governed equations [11] are presented as equation (1).

The preliminary values of true anomaly ( $\theta$ ), longitude of the deorbit Ascending Node $(\psi)$ and deorbit velocity $\left(V_{\text {deorb }}\right)$ are recognized as the optimization parameters but inclination

(i) is constant and equals to $55^{\circ}$. Thrust is available during the deorbit maneuver and it is considered that thrust vector always acts apposite the velocity vector.

$\dot{\overrightarrow{\mathrm{V}}}_{\mathrm{OI}}=\left[\begin{array}{c}\dot{\mathrm{V}}_{\mathrm{x}} \\ \dot{\mathrm{V}}_{\mathrm{y}} \\ \dot{\mathrm{V}}_{\mathrm{z}}\end{array}\right]_{\mathrm{OI}}=\left[\begin{array}{c}\left(-(\mathrm{D}+\mathrm{T}) / \mathrm{M}_{\mathrm{RV}}\right) \cos (\beta-\gamma)-\mathrm{g} \sin (\beta) \\ \mathrm{g} \cos (\beta)-(\mathrm{D}+\mathrm{T}) / \mathrm{M}_{\mathrm{RV}} \sin (\beta-\gamma) \\ 0\end{array}\right]$

$\dot{\overrightarrow{\mathrm{R}}}_{\mathrm{OI}}=\overrightarrow{\mathrm{V}}_{\mathrm{OI}}$

$\overrightarrow{\mathrm{R}}_{\mathrm{e}}=\left[\mathrm{R}_{\mathrm{x}}, \mathrm{R}_{\mathrm{y}}, \mathrm{R}_{\mathrm{z}}\right]=\mathrm{C}_{\mathrm{EI}}^{\mathrm{e}} \mathrm{CII}_{\mathrm{OI}}^{\mathrm{EI}} \overrightarrow{\mathrm{R}}_{\mathrm{OI}} \quad, \quad \gamma=\frac{\pi}{2}-\cos ^{-1}\left(\frac{\overrightarrow{\mathrm{R}}_{\mathrm{OI}} \cdot\left(-\overrightarrow{\mathrm{V}}_{\mathrm{OI}}\right)}{\left|\overrightarrow{\mathrm{R}}_{\mathrm{OI}}\right|\left|\overrightarrow{\mathrm{V}}_{\mathrm{OI}}\right|}\right)$

$\beta=\tan ^{-1}\left(\frac{\mathrm{R}_{\mathrm{x}}^{\mathrm{ol}}}{\mathrm{R}_{\mathrm{y}}^{\mathrm{ol}}}\right)$

$\mathrm{C}_{\mathrm{OI}}^{\mathrm{EI}}=\left[\begin{array}{ccc}-\mathrm{c}(\theta) \mathrm{s}(\psi) \mathrm{c}(\mathrm{i})-\mathrm{c}(\psi) \mathrm{s}(\theta) & \mathrm{s}(\psi) \mathrm{s}(\theta) \mathrm{c}(\mathrm{i})-\mathrm{c}(\theta) \mathrm{c}(\psi) & \mathrm{s}(\psi) \mathrm{s}(\mathrm{i}) \\ \mathrm{c}(\mathrm{i}) \mathrm{c}(\theta) \mathrm{c}(\psi)-\mathrm{s}(\theta) \mathrm{s}(\psi) & -\mathrm{c}(\psi) \mathrm{s}(\theta) \mathrm{c}(\mathrm{i})-\mathrm{c}(\theta) \mathrm{s}(\psi) & -\mathrm{c}(\psi) \mathrm{s}(\mathrm{i}) \\ \mathrm{c}(\theta) \mathrm{s}(\mathrm{i}) & -\mathrm{s}(\mathrm{i}) \mathrm{s}(\theta) & \mathrm{c}(\mathrm{i})\end{array}\right]$

$\mathrm{C}_{\mathrm{EI}}^{\mathrm{e}}(\mathrm{t})=\left[\begin{array}{ccc}\cos \left(\omega_{\mathrm{e}} \mathrm{t}\right) & -\sin \left(\omega_{\mathrm{e}} \mathrm{t}\right) & 0 \\ \sin \left(\omega_{\mathrm{e}} \mathrm{t}\right) & \cos \left(\omega_{\mathrm{e}} \mathrm{t}\right) & 0 \\ 0 & 0 & 1\end{array}\right]$
The trajectory discipline is shown at figure (2).

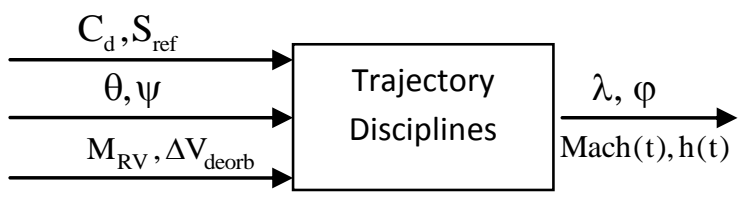

Figure-2: Flow data of trajectory Simulation

\section{HEAT SHIELD AND THERMAL PROTECTION SYSTEM}

Ablative thermal protection systems (TPS) are the natural selection for this kind of unguided trajectories. In fact, ablative materials can accommodate heating rates and heat loads through the reentry trajectories. The ablative heat shield functions by lifting the hot shock layer gas away from the heat shield's outer wall (creating a cooler boundary layer) through blowing. The overall process of reducing the heat flux experienced by the heat shield's outer wall is called blockage. Ablation causes the TPS layer to char, melt, and sublime through the process of pyrolysis. Carbon phenolic is a very effective ablative material and so it is selected for using in this paper. Total heat flux function is presented as equation (2) and it should be lower than payload tolerance which is equal to $11750(\mathrm{Kj})$ in this paper.

$$
\begin{aligned}
& Q_{\text {total }}=\int_{0}^{t_{f}}\left(\dot{q}_{N} S_{N}+\dot{q}_{m} S_{m}+\dot{q}_{s} S_{s}+\dot{q}_{b} S_{r e f}\right) d t \\
& \dot{q}_{i}=\rho_{T P S} S_{i} \Delta x_{i} C_{\text {Albative }} \\
& \rho_{T P S}=2740\left(\frac{\mathrm{kg}}{\mathrm{m}^{3}}\right)
\end{aligned}
$$

Finally, the TPS thickness $\left(\Delta x_{i}\right)$ introduced as the optimization parameters. It should be noted that lateral surfaces, TPS kind and aerodynamic coefficients are known. Data flow of the TPS modeling has been shown at figure (3). 


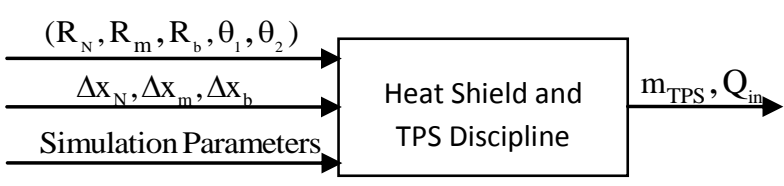

Figure-3: Flow data of structural modeling

\section{PROPULSION MODELING}

RV needs to decrease the orbital velocity to return. Deorbit phase involves in propulsion system activity and fuel consumption. The required propellant mass for deorbit phase is shown as equation (3).

$$
\left\{\begin{array}{l}
m_{\text {prop }}=\left(1-\mu_{e}\right) M_{\text {total }} \\
\mu_{e}=e^{\frac{-\Delta V_{\text {deort }}}{I^{s p . g_{0}}}} \\
\Delta V_{\text {deorb }}=\int_{0}^{t_{f}} \frac{T}{M_{\text {Total }} g_{0}-\dot{m}_{\text {nozzel }}} d t \\
T=I_{\text {sp }} g_{0} \dot{m}_{\text {nozzel }} \\
V_{\text {Propellant }} \leq 1.42\left(m^{3}\right) \\
t_{f}=\frac{m_{\text {propellant }}}{\dot{m}_{\text {nozzel }}}
\end{array}\right.
$$

Four kinds of propulsion systems can be used for deorbit phase including bi-propellant, solid propellant and monopropellant propulsion system. For the low payloads, monopropellant propulsion system with separated supply gas tank can be considered. In this paper, green hydrogen peroxide mono-propellant is selected. Temperature and physical-chemical property of production of decomposition are strongly correlated to the concentration of $\mathrm{H}_{2} \mathrm{O}_{2}$. Equation

(4) shows the governing relation [12]

$$
\varphi \mathrm{H}_{2} \mathrm{O}_{2}+(1-\varphi) \mathrm{H}_{2} \mathrm{O} \rightarrow \mathrm{H}_{2} \mathrm{O}+\frac{\varphi}{2} \mathrm{O}_{2}
$$

Where, $\varphi$ is molar percentage of the concentration of $\mathrm{H}_{2} \mathrm{O}_{2}$. Equation (4) shows that only the superheated steam and oxygen are released from decomposition process. It means that no other toxic gas is released to the air. Table -1 presents properties of $\mathrm{H}_{2} \mathrm{O}_{2}$ decomposition products [12].

Table 1 Physical-chemical Property of $\mathrm{H}_{2} \mathrm{O}_{2}$

\begin{tabular}{|c|c|c|c|c|}
\hline $\begin{array}{c}\text { concentration of } \\
\mathbf{H}_{\mathbf{2}} \mathbf{O}_{\mathbf{2}}\end{array}$ & $\mathbf{8 5 \%}$ & $\mathbf{9 0 \%}$ & $\mathbf{9 5 \%}$ & $\mathbf{9 8 \%}$ \\
\hline Adiabatic Temp.(k) & 907 & 1029 & 1151 & 1225 \\
\hline Molecular mass & 21.83 & 22.10 & 22.39 & 22.56 \\
\hline$\gamma$ & 1.2751 & 1.2647 & 1.2557 & 1.2509 \\
\hline Density $\left(\frac{\mathrm{kg}}{\mathrm{m}^{3}}\right)$ & 1340 & 1360 & 1380 & 1392 \\
\hline
\end{tabular}

\subsection{Propellant Tank Modeling}

Spherical tanks demand a lower thickness in comparison with cylindrical types, but cylindrical tanks demand lower diameter and simpler production procedures. The propellant tank configuration is limited by maximum permitted diameter. If spherical tank cannot provide the required volume, cylindrical tank with maximum permitted diameter and suitable length is considered. Equation (5) presents the configuration selection method.

$$
\begin{aligned}
& \text { if } V_{\text {Tank }} \leq \frac{4 \pi R_{\max }^{3}}{3} \Rightarrow R_{\text {Tank }}=\sqrt[3]{\frac{3 V_{\text {Tank }}}{4 \pi}} \\
& \text { if } V_{\text {Tank }}>\frac{4 \pi R_{\text {max }}^{3}}{3} \Rightarrow\left\{\begin{array}{l}
R_{\text {Tank }}=R_{\max } \\
L_{c y l}=\frac{\left(V_{\text {Tank }}-\frac{4 \pi R_{\max }^{3}}{3}\right)}{\pi R_{\max }^{2}}
\end{array}\right.
\end{aligned}
$$

The mass of propellant tank is related to tank pressure, tank configuration, filling factor, propellant concentration and propellant mass. The mass of propellant tank is estimated by equation (6).

$M_{\text {Tank }}=\left(4 p R_{\text {Tank }}^{2}+2 p R_{\text {Tank }} L_{c y l}\right) r_{\text {str }} d_{\text {Tank }}$

$d_{\text {Tank }}^{\text {Sph }}=\frac{n_{S . F}}{2 s_{\text {str }}} P_{\text {Tank }} R_{\text {Tank }} \quad, \quad d_{\text {Tank }}^{c y l}=2 d_{\text {Tank }}^{\text {Sph }}$

Aluminum or titanium structures are generally used for space application but lower cost of aluminum provides more interest. Input-output data of propellant tank model has been presented in figure (4).

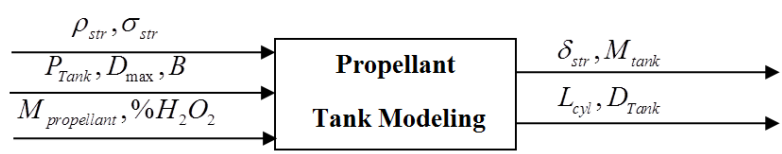

Figure 4: Flow data of propellant tank modeling

\subsection{Pressurized Feeding Modeling}

Feeding subsystem should produce the safe continuous or discontinuous propellant flow to the catalyst bed (thruster). A separate gas supply pressurizes the propellant tank to force $\mathrm{H}_{2} \mathrm{O}_{2}$ flows on the catalyst bed. The most part of the feeding subsystem mass including gas tank and pressurizer gas [12]. The required radius of gas tank is derived by equation (7).

$$
\begin{aligned}
& V_{\text {Press.Tank }}=\frac{R T\left(M_{P . G}(\text { total })-M_{\text {Press.Gas }}^{(t=0)}\right.}{P_{\max }} \\
& R_{\text {Press.Tank }}=\sqrt[3]{\frac{3 V_{\text {Press.Tank }}^{(t=0)}}{4 \pi}}
\end{aligned}
$$

Titanium structure is selected for pressurized tank. The feeding subsystem mass is estimated by equation (8).

$$
\begin{aligned}
& M_{\text {feeding }}=M_{P . T}+M_{P . g} \\
& M_{P . T}=4 \pi R_{P . T}^{2} \rho_{s t r} \delta_{P . T} \\
& \delta_{P . T}=\frac{n_{S . F}}{2 \sigma_{s t r}} P_{\max } R_{P . T} \\
& M_{P . g}=\frac{4 P_{\max }}{3 R_{g a s} T_{P . g}} \pi R_{P . T}^{3} \rho_{s t r}
\end{aligned}
$$

Flow data of the feeding subsystem mode is presented in figure (5).

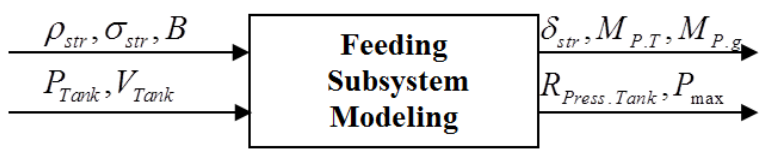

Figure 5: Input-Output flow data of pressurized feeding 


\subsection{Thruster Modeling}

Thruster consists of catalyst, catalyst bed and nozzle. Thrust level and Isp introduce the thruster's size and thruster's performance respectively. Equation (9) presents the governing relation [12]. Steel structure is commonly used for the thruster.

$$
\begin{aligned}
& T_{v a c}=\frac{A^{*} P_{e}\left(\gamma M_{e}^{2}+1\right)}{M_{e}^{2}}\left[\frac{2}{\gamma+1}\left(1+\frac{\gamma-1}{2} M_{e}^{2}\right)\right]^{\frac{\gamma+1}{2(\gamma-1)}} \\
& I s p_{v a c}=\frac{T_{v a c}}{\dot{m} g_{0}}
\end{aligned}
$$

\subsubsection{Catalyst}

The most significant technology challenge of hydrogen peroxide monopropellant thrusters is the development of new effective catalyst. Nowadays, the most used catalyst materials for $\mathrm{H}_{2} \mathrm{O}_{2}$ are metallic silver, $\mathrm{MnO}_{2}$ and $\mathrm{Mn}_{2} \mathrm{O}_{3}$ and aluminadeposited platinum. Most important characteristics of the catalyst are ability to decompose the propellant flow (named catalyst bed loading) and longer activity. Table- 2 shows some physical parameters of the considered catalyst [12].

Table 2. Some properties of selected catalyst

\begin{tabular}{|c|c|}
\hline Residence Time $(\mathbf{m}-\mathbf{s e c})$ & 2.7 \\
\hline$L_{f}^{\max }\left(P_{P}\right)$ & $: 200$ \\
\hline $\operatorname{Density}\left(\frac{\mathrm{kg}}{\mathrm{m}^{3}}\right)$ & $: 300$ \\
\hline $\mathrm{D} t^{\max }(\mathrm{sec})$ & $<500$ \\
\hline
\end{tabular}

\subsubsection{Catalyst bed}

Length and diameter of the catalyst bed introduce the geometry. Maximum pressure of the catalyst bed specifies required thickness of thruster. Important event in catalyst bed is pressure drop during the injection and movement through the catalyst bed. Equation (10) shows the relation between propellant tank pressure and thruster pressure [12].

$P_{\text {Tank }}=P_{T h}+\Delta P_{c t l}+\Delta P_{i n j}+\Delta P_{p i p e}$

$\Delta P_{\text {pipe }}=0.5$ bar

$\Delta P_{i n j}=0.15\left(P_{\text {Tank }}-\Delta P_{p i p e}\right)$

$\Delta P_{c t l}($ bar $)=\frac{6}{75} L_{c t l}(m m)$

$L_{c t l}=1.2 \bar{V} t_{\text {residence }}$

$\bar{V} \approx 1.5 V_{i n j}$

$V_{i n j}^{2}-\frac{C d_{i n j}^{2} P_{T h}}{2 \rho_{\mathrm{H}_{2} \mathrm{O}_{2}}}-\frac{120 t_{\text {residence }} C d_{i n j}^{2}}{\rho_{\mathrm{H}_{2} \mathrm{O}_{2}}} V_{i n j}=0$

\subsubsection{Divergent-Convergent Nozzle}

High pressure and temperature flow exits from the catalyst bed and enters the D-C nozzle. It changes the potential energy to the kinetic energy. The conical nozzle is selected for conceptual design phase. The nozzle geometry is illustrated in figure (6).

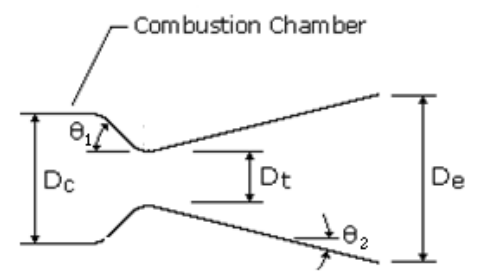

Figure 6: Nozzle geometry parameters

Equation (11) presents the relation of geometry parameters and thermodynamic parameters.

$\theta_{1}=45^{\circ} \quad, \quad \theta_{2}=15^{\circ}$

$A^{*}=\frac{\dot{m} \sqrt{\frac{T_{c} R}{\gamma}}}{P_{T h}}\left(1+\frac{\gamma-1}{2}\right)^{\frac{\gamma+1}{2(\gamma-1)}}$

$A_{e}=\frac{A^{*}}{M_{e}}\left[\frac{2}{\gamma+1}\left(1+\frac{\gamma-1}{2} M_{e}^{2}\right)\right]^{\frac{\gamma+1}{2(\gamma-1)}}=A^{*} Z_{\text {expansion }}$

$L_{d i v}=\frac{R_{c t l}-R_{t}}{\tan \theta_{1}} \quad, \quad L_{d i v}=\frac{R_{e}-R_{t}}{\tan \theta_{2}}$

Total mass of the thruster (catalyst, catalyst bed and nozzle) is estimated from equation (12).

$$
\begin{aligned}
& M_{\text {Thruster }}=M_{\text {Comb }}+M_{\text {Nozzle }}+M_{c a t} \\
& M_{\text {Comb }}=\left(2 p R_{c t l} L_{c t l}+p R_{c t l}^{2}\right) d_{C o m b} r_{s t r} \\
& M_{\text {Nozzle }}=\left[\frac{p}{\tan q_{1}}\left(R_{c t l}^{2}-R_{t}^{2}\right)+\frac{p}{\tan q_{2}}\left(R_{e}^{2}-R_{t}^{2}\right)\right] d_{C o m b} r_{s t r} \\
& d_{\text {Comb }}=\frac{n_{S . F}}{s_{s t r}}\left(\frac{p P_{T h} R_{c t l}^{2}+T}{p R_{c t l}}\right) \\
& M_{c a t}=r_{c t l} A_{c} L_{c t l}
\end{aligned}
$$

Finally, flow data of the thruster model is presented in figure (7).

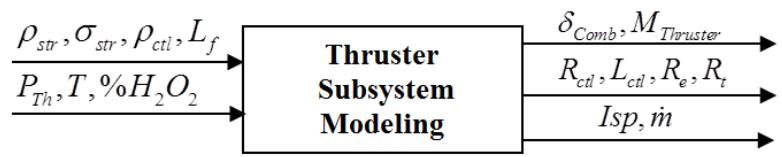

Figure 7: Input-Output flow data of thruster

\section{THE REENTRY MISSION \\ OPTIMIZATION ALGORITHM}

For the current study, the genetic algorithm (GA) is chosen as system optimizer. One of the main benefits of using a GA is the fact that the algorithm can find the global optimum and uses a population of guesses that are random and spread throughout the search space. Powerful operators such as selection, crossover, and mutation help direct members of each population toward the desired goal of the problem. The total mass of RV is selected as the cost function in the system level optimizer. This function is presented as equation (4).

$$
\begin{aligned}
& \mathrm{M}_{\text {Total }}=\mathrm{m}_{\text {prop }}+\mathrm{m}_{\mathrm{TPS}}+\mathrm{m}_{\text {str }}+\mathrm{m}_{\text {payload }}+\mathrm{m}_{\text {tank }} \\
& \mathrm{m}_{\text {payload }}=250(\mathrm{~kg})
\end{aligned}
$$

GA is applied to optimize the deorbit parameters. Finally, following input parameters are adjusted in GA optimization

- Population size: 250

- Number of generations: 50 


\section{- Mutation probability: $0.4 \quad$ (Floating point) \\ - Cross-over probability: 0.6 (Floating point) \\ - Mutation type: Adaptive feasible \\ - Cross-over type: Stochastic uniform \\ - Selection: Uniform}

Finding the feasible set of parameters is one of the reasons of long processing time. Some points are understood meanwhile the optimization help to decrease the processing time. These points are presented as follow.

1. Ablative heat shield thicknesses $\left(\Delta x_{i}\right)$ depend on trajectory parameters and it can be said that the thicknesses are designed for every path and aerodynamic configuration. Increase in the heat shield thicknesses, more than required value, leads to increase in TPS mass and decrease in the total absorbed heat flux. Therefore, the optimum selection is minimum heat shield thicknesses with satisfying the heat flux constraint.

2. Deorbit velocity has major effect on the trajectory, TPS mass and propellant mass. Total flight time is a function of the deorbit velocity or $\Delta V_{\text {deorb }}$. Increase in $\Delta V_{\text {deorb }}$ leads to shorter flight time and thicker heat shields but it increases the orbital plane earth range. $\psi$ and $\theta$ are independent parameters because they are coupled by orbital movement and can be presented as equation (5).

$\psi=-\omega_{e} t+\psi_{0}$

$\theta=\omega_{\theta} t+\theta_{0}$

$\omega_{e}=7.29 \times 10^{-5}$

$\omega_{\theta}=\frac{\left|\overrightarrow{\mathrm{V}}_{\mathrm{oI}}\right|}{\left|\overrightarrow{\mathrm{R}}_{\mathrm{oI}}\right|}=0.0012$

$\Rightarrow \psi=-\frac{\omega_{e}}{\omega_{\theta}} \theta+\frac{\omega_{e}}{\omega_{\theta}} \theta_{0}+\psi_{0}$

$\Rightarrow \psi(\mathrm{rad})=-0.06075 \theta(\mathrm{rad})+4.7758$

3. The mission error is decreased by decrease in the propellant mass. Decrease in the propellant mass leads to increase in the deorbit velocity. Increase in the deorbit velocity leads to increase in the TPS mass. The variations of the propellant mass and TPS mass are complex and hardly can be estimated. Multidisciplinary design optimization based on AAO can be used as sensitive analyzer so it derives the best solution in shorter elapsed time.

Considering mentioned points, it leads to derive the feasible parameter sets and elapsed time of multidisciplinary optimization decrease. The optimum designs of the deorbit mission are derived for single discipline and multidisciplinary disciplines optimization and they are compared in table- 1 .

Table 3 Optimum reentry mission results

\begin{tabular}{|c|c|c|c|}
\hline Discipline Optimization & TPS only & $\begin{array}{c}\text { Propulsion } \\
\text { only }\end{array}$ & MDO \\
\hline$\Delta V_{\text {deorb }}(\mathrm{m} / \mathrm{s})$ & 911.4 & 209.6 & 410.2 \\
\hline Thrust $(\mathrm{N})$ & 104 & 944 & 910 \\
\hline TPS mass $(\mathrm{kg})$ & 915.2 & 1272.2 & 1050.7 \\
\hline Propellant mass $(\mathrm{kg})$ & 549.2 & 126.9 & 232.2 \\
\hline Total mass $(\mathrm{kg})$ & 2359.1 & 2139.0 & 2063.1 \\
\hline
\end{tabular}

Comparison of the results shows that MDO technique is more suitable for complex design problems. Lower total mass $(\sim 3.6 \%)$ confirms the performance of MDO technique in comparison with single discipline design optimization.

\section{SUMMARY AND CONCLUSION}

Optimization of the deorbit mission was the goal of this paper. TPS mass and propellant mass have major effect on the mass of reentry vehicle which is a good approximate of reentry mission cost. MDO technique was used to solve the conflicts between trajectory, TPS and propulsion disciplines and find the optimum deorbit mission considering some paths, configuration and thermal constrains. Every discipline was developed base on the AAO framework. GA was adjusted for this problem and the results were introduced for minimum propulsion mass, minimum TPS mass and minimum total mass separately. The comparison of the results confirms the performance of MDO technique to decrease close to $3.6 \%$ mass of reentry mission. As future works, solid propellant or bipropellant propulsion system can be considered. In addition, other system optimizer such as SQP or hybrid optimizer such as GA-SQP can be applied.

\section{REFERENCES}

[1] Multidisciplinary Design Optimization of RLV Reentry Trajectory. Chen Gang, Xu Min and et al.. , AIAA/CIRA 13th International Space Planes and Hypersonics Systems and Technologies, 2005. AIAA 2005-3270.

[2] optimal skips entry into atmosphere with minimum heat. Istratie, Vasile. Austin, Texas : AIAA Atmospheric Flight AIAA Atmospheric Flight, 11-14 August, 2003.

[3] Aerodynamic stability of reentry heat shield shapes for a crew exploration vehicle. J.E. Johnson and et.al., No.4, Journal of Spacecraft and Rockets, July-Aug 2006, Vol. 43.

[4] Hypersonic Aerothermodynamics/Aerothermoelastics Methodology for Reusable Launch Vehicles/TPS Design and Analysis. Liu D., Chen P., Tang L., and Chang K., 41st Aerospace Sciences Meeting and Exhibit, Reno, Nevada, Jan. 6-9, 2003. AIAA-2003-897.

[5] Trajectory and Thermal Protection System Design for Reusable Launch Vehicles. McGuire M., Gage P., Galloway E., Huynh L., 10th AIAA/ISSMO Multidisciplinary Analysis and Optimization Conference, Albany, New York, Aug. 30-1, 2004. AIAA Paper 20044490.

[6] Aerodynamic Shape Optimization of a Re-entry Capsule. Rajesh Kumar Arora, Pradeep Kumar., AIAA Atmospheric Flight Mechanics Conference and Exhibit, Austin, Texas, 11 - 14 August 2003.

[7] Multidisciplinary design optimization of a re-entry vehicle shape and trajectory. M. Tava, S. Suzuki, 2001. AIAA-2001-1920.

[8] Multidisciplinary design optimization of a reentry vehicle using genetic algorithm. M. Nosratollahi, M. Mortazavi, A. Adami, M. Hosseini, Aircraft Engineering and Aerospace Technology, 2010, Vol. 82, No:3, pp.194203.

[9] Multidisciplinary Design Optimization of a Controllable Reentry Capsule for minimum Landing velocity. M. Nosratolahi, M. Hoseini, A. Adami. 51st AIAA/ASME/ ASCE/AHS/ASC Structures, Structural Dynamics, and 
Materials Conference, Florida , 12-15 April 2010. AIAA-2010-3009.

[10] Multidisciplinary Design Optimization of a Manned Reentry Mission Considering Trajectory and Aerodynamic Configuration. Amirhossein Adami, Mehdi Mortazavi, Mehran Nosratollahi, Majid Hosseini, International Conference on Recent Advances in Space Technologies, Stanbul, Turkye, 09-11 June 2011.

[11] Adami, Amirhossein. Multidicsiplinary design optimization of Reentry Vehicle considering Guidance
Algorithm. Aerospace Department. Tehran, Iran , Amirkabir University of Technology, 2014. Ph.d thesis.

[12] Multidisciplinary Design Optimization of Hydrogen Peroxide Monopropellant Propulsion System using GA and SQP. Adami Amirhossein, Mortazavi Mahdi and Nosratollahi Mehran. 9, March 2015, International Journal of Computer Applications, Vol. 113, pp. pp.1421. 